\title{
Modal control of an HV DC system for the damping of subsynchronous oscillations
}

\author{
Y.-Y. Hsu \\ L. Wang
}

Indexing terms: Subsynchronous resonance, HV DC link, Modal control, Control theory

Abstract: A novel control scheme is presented for the design of a supplementary subsynchronous damping controller (SSDC) equipped at the rectifier end of an HV DC link that is connected in parallel with an existing resonating AC line. To stabilise the unstable subsynchronous resonance (SSR) modes, an approach based on modal control theory is developed for the design of the SSDC. Since the subsynchronous oscillations resulting from high levels of compensation on the AC line can be effectively damped out, the power transfer capability of the AC line can be enhanced by the proposed SSDC. In addition, the SSDC can also be used to increase the damping of torsional interaction between the HV DC link and turbine generator, thereby qualifying the HV DC link equipped with the proposed SSDC as an alternative to a new, second $\mathrm{AC}$ line where long distances of transmission may economically justify the use of an HV DC line.

\section{List of symbols}

General

$A, B, C=$ system matrix

$\omega, \theta=$ rotor speed and rotor angle

$\delta \quad=$ generator torque angle

$U_{E}, U_{R}=$ auxiliary input signals of exciter and rectifier

$E_{F D} \quad=$ per unit output voltage of exciter

$V_{R E} \quad=$ voltage regulator output

$a \quad=$ actuator signal

$g \quad=$ governor opening signal

$F \quad=$ fractions of mechanical torque distribution

$e_{c}=\mathrm{AC}$ line capacitor voltage

$\stackrel{c}{\infty}_{\infty} \quad=$ infinite busbar voltage

$\alpha=$ rectifier delay angle

$\gamma \quad=$ inverter extinction angle

$X_{C R}, X_{C I}=$ commutating chock reactances of rectifier and inverter

$V_{d R}, V_{q R}=$ p.u. RMS rectifier voltages refer to $d_{R} q_{R}$ axis

$I_{d R}, I_{q R}=$ p.u. RMS rectifier currents refer to $d_{R} q_{R}$ axis

$V_{d}, V_{q}=$ p.u. RMS voltages refer to $d q$-axis

$I_{d}, I_{q}^{q} \quad=$ p.u. RMS currents refer to $d q$-axis

$\delta_{R} \quad=$ angle by which $q_{R}$-axis lags $q$-axis

$\phi_{R} \quad=$ angle between $V_{q R}$ and $I_{R}$

$V_{R}, V_{I}=$ p.u. rectifier and inverter voltages

$I_{R}, I_{I}=$ p.u. rectifier and inverter currents

Paper 6404C (P9), received 4th May 1988

The authors are with the Department of Electrical Engineering, National Taiwan University, Taipei, Taiwan, Republic of China

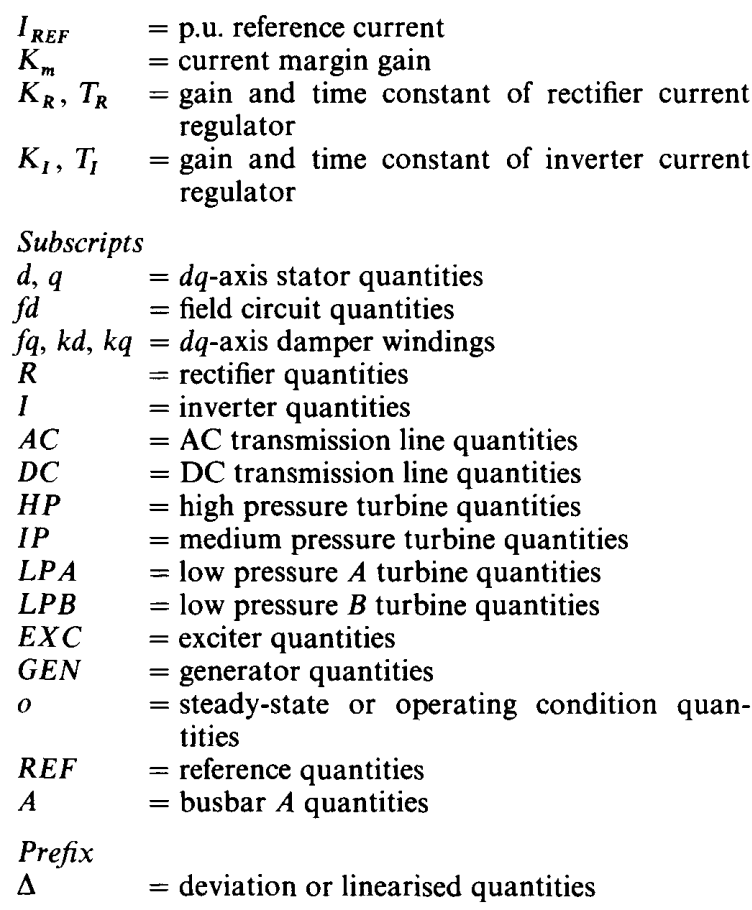

\section{Introduction}

Since the torsional interaction between the HV DC link and the turbine generator at Square Butte in North Dakota was first reported in 1980 [1], several studies have been made to identify the cause of interaction and to devise countermeasures for these subsynchronous oscillations [2-7].

Experience with the Square Butte DC link indicated that the rectifier current control loop and the frequency sensitive power control (FSPC), that was designed to damp interarea oscillations, could cause an undamped $11.5 \mathrm{~Hz}$ oscillation under certain operating conditions $[1,3]$. The damping for this torsional oscillation was improved by an adjustment to the transfer function of the rectifier current control loop and the incorporation of a $11.5 \mathrm{~Hz}$ notch filter into the FSPC. The subsequent tests on a similar system configuration, the CU HV DC link, showed that there was no risk of undamped subsynchronous oscillations owing to the higher first torsional mode frequency of $19 \mathrm{~Hz}$ on the Coal Creek Unit $[2,4,5]$. In view of the potential torsional oscillations associated with an HV DC link, a reasearch project was sponsored by the Electric Power Research Institute [6]

IEE PROCEEDINGS, Vol. 136, Pt.C, No. 2, MARCH 1989 
to develop a supplementary subsynchronous damping controller (SSDC) for the damping of these oscillations. With its parameters tuned by using the Bode plot (gain plot and phase plot), the designed SSDC could provide positive damping for the subsynchronous oscillations under some operating conditions.

Recently, Harley and Balda [7] employed a different approach for the design of an SSDC. The eigenvalues of the torsional modes were scanned as the parameters of the SSDC were varied. Then a suitable set of parameters were chosen by a scrutiny of the resulting torsional mode eigenvalues. This trial-and-error approach suffers from a major drawback of requiring a great amount of computational time because there are several parameters to be determined simultaneously, and the highly timeconsuming work of computing system eigenvalues must be repeated over and over again, as these multidimensional unknown parameters are varied.

The purpose of this paper is to present a systematic approach for the design of an SSDC. The parameters of the SSDC are determined by using a modern control theory called modal control $[8,9]$. To investigate the effect of the proposed SSDC on the torsional interaction between the HV DC line and the turbine generator as well as the SSR modes on an existing capacitorcompensated AC line, the SSDC is equipped at the rectifier end of an HV DC link connected in parallel with the AC line. Sensitivity studies with regard to line flow, delay angle and the degree of series compensation on the AC line reveal that the proposed SSDC is insensitive in that it can provide good damping effects for the subsynchronous oscillations over a wide range of operating conditions. In addition to these frequency-domain eigenvalue analyses which deal exclusively with the linearised system, time domain simulations using a nonlinear system model are also performed to demonstrate the effectiveness of the proposed SSDC under disturbance conditions.

\section{System model}

The system considered is the IEEE First Benchmark Model [10] with an HV DC link included, as shown in Fig. 1.

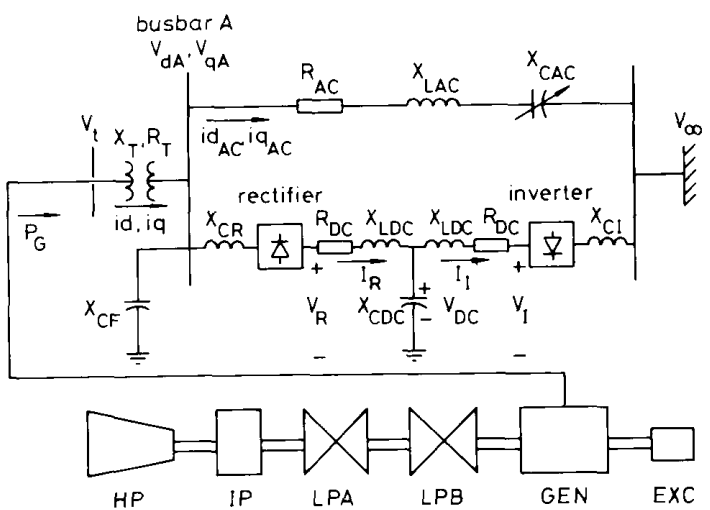

Fig. 1 IEEE first benchmark model with an HV DC link included

An equivalent $T$ network [11], with two series impedances placed on both sides of a lumped charging capacitance that is put at the midpoint of the line, is used as the model for the DC line. The $d q$-axis representations of DC transmission line equations are described in Appen- dix 9.1. Appendix 9.2 contains the equations for the turbine torques and speed governor system.

The system data using the same base values as those adopted in Reference 10 are given in Table 1 [12-16].

Table 1: System data

\begin{tabular}{|c|c|c|c|}
\hline \multicolumn{4}{|c|}{ Mass-spring parameters } \\
\hline Mass & Shaft & $\begin{array}{l}\text { Inertia } \\
\mathrm{HI}, \mathrm{s}\end{array}$ & $\begin{array}{l}\text { Spring } \\
\text { constant } \\
\text { K, p.u. torque/rad }\end{array}$ \\
\hline$H P$ & & 0.092897 & 19303 \\
\hline IP & $\angle P-L P A$ & 0.155589 & $\begin{array}{l}19.303 \\
34.929\end{array}$ \\
\hline$\angle P A$ & $\angle P A-L P B$ & 0.858670 & 52.038 \\
\hline$\angle P B$ & LPB-GEN & 0.884215 & 70.858 \\
\hline GEN & & 0.868495 & 2.822 \\
\hline EXC & & 0.0342165 & \\
\hline
\end{tabular}

Turbine torque and speed governor system

$\begin{array}{lll}T_{C H}=0.3 \mathrm{~s} & T_{P H}=7.0 \mathrm{~s} & T_{C O}=0.2 \mathrm{~s} \\ K_{G}=25.0 & T_{S A}=0.2 \mathrm{~s} & T_{S M}=0.3 \mathrm{~s} \\ F_{H P}=30 \% & F_{I P}=26 \% & F_{L P A}=22 \% \\ F_{L P B}=22 \% & & \end{array}$

Synchronous generator, p.u.

$X_{d}=1.790 \quad x_{t d}=0.04 \quad x_{k d}=0.006 \quad x_{\text {sdd }}=1.660$

$\begin{array}{llll}X_{d}=1.710 & X_{t q}=0.04 & X_{k d}=0.006 & X_{\text {ad }}=1.660 \\ X_{q}=0.115 & X_{k q}=0.245 & X_{a q}=1.580\end{array}$

$R_{a}=0.0015 \quad R_{f d}=0.001 \quad R_{k d}=0.0037 \quad R_{f q}=0.0053$

$R_{k q}=0.0182$

Exciter and voltage regulator

$K_{A}=50.0 \quad E_{F D \text { max }}=7.3$ p.u. $\quad U_{E \text { max }}=0.12$ p.u.

$T_{A}^{A}=0.01 \mathrm{~s} \quad E_{F D \text { min }}=-7.3$ p.u. $\quad U_{E \text { min }}=-0.12$ p.u.

Transformer and ACDC transmission line, $p . u$.

$\begin{array}{llll}R_{T}=0.01 & X_{T}=0.14 & R_{A C}=0.02 & X_{L A C}=0.56 \\ R_{D C}=0.05 & X_{L D C}=0.920 & X_{C D C}=8.333 & X_{C C}=10.0\end{array}$

Rectifier and inverter current regulator

$\begin{array}{llll}K_{R}=5.0 & T_{R}=0.01 \mathrm{~s} & K_{L}=5.0 & T_{1}=0.01 \mathrm{~s} \\ a_{R \text { max }}=20^{\circ} & a_{R \text { min }}=10^{\circ} & U_{R \max }=0.1 \text { p.u. } & U_{R \text { min }}=-0.1 \text { p.u. } \\ K_{m}=0.9 & & & \end{array}$

Operating conditions

$V_{t o}=1.05$ p.u.

$x_{C A C}^{t o} / X_{L A C}=50 \%$

$X_{C A C}=0.4064$ p.u.
$P_{A C O}=0.9349$ p.u.

$V_{R O}^{A C O}=0.9349$ p.u.

$V_{d A O}=0.7179$ p.u.

$i_{\text {वACo }}^{d A O}=0.1877$ p.u.

$i_{d o}^{\text {GACo }}=1.5022$ p.u.

system frequency $=60 \mathrm{~Hz}$

$$
\begin{aligned}
& P_{G o}=0.9 \text { p.u. } \quad P F=0.9 \text { lagging } \\
& a_{R o}=18^{\circ} \quad Y_{1 O}=15^{\circ} \\
& \begin{array}{ll}
P_{D C O}=0.4846 \text { p.u. } & \left|V_{A O}\right|=0.9901 \text { p.u. }
\end{array} \\
& J_{R o}=0.5183 \text { p.u. } \quad V_{\infty}=0.9212 \text { p.u. } \\
& V_{\text {aAo }}=0.6819 \text { p.u. } \quad i_{\text {dACO }}=0.8021 \text { p.u. } \\
& V_{D C O}=0.9090 \text { p.u. } \quad l_{1 o}=0.5183 \text { p.u. } \\
& i_{\text {go }}=0.6815 \text { p.u. }
\end{aligned}
$$

Under the operating condition of $V_{t}=1.05$ p.u., $P_{G}=$ 0.9 p.u., power factor $=0.9$ lagging, $\alpha_{R}=18^{\circ}, \gamma_{I}=15^{\circ}$ and $X_{C A C} / X_{L A C}=50 \%$, the eigenvalues for an AC system and a parallel ACDC system without an SSDC for the HV DC link are listed in Table 2.

It can be observed that, without an SSDC, the inclusion of a parallel HV DC link cannot damp out the subsynchronous oscillations on an existing capacitor-compensated line.

To see how the dampings of the torsional modes vary with the degree of compensation, the real parts of the eigenvalues for modes $0-5$ of the ACDC system as a function of the ratio $X_{C A C} / X_{L A C}$ are depicted in Fig. 2 .

It is evident from Table 2 and Fig. 2 that all SSR modes, except modes 2 and 5 , may become unstable since, under certain operating conditions, the eigenvalues associated with these modes may have positive real parts. 


\begin{tabular}{|c|c|c|c|c|}
\hline \multicolumn{3}{|r|}{ AC system } & \multirow{2}{*}{$\begin{array}{l}\text { ACDC system } \\
\text { without an SSDC } \\
-0.1818 \pm \mathrm{j} 298.18 \\
0.0171 \pm \mathrm{j} 202.72 \\
0.4245 \pm \mathrm{j} 160.43 \\
-0.6399 \pm \mathrm{j} 127.08 \\
-0.1237 \pm \mathrm{j} 99.51 \\
-0.3325 \pm \mathrm{j} 10.18\end{array}$} & \multirow{2}{*}{ 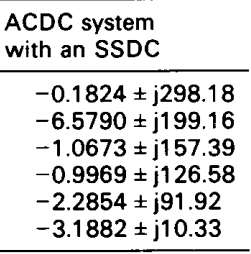 } \\
\hline $\begin{array}{l}\text { Shaft } \\
\text { modes }\end{array}$ & $\begin{array}{l}\text { (5) } \lambda_{H P} \\
\text { (4) } \lambda_{\text {IP }} \\
\text { (3) } \lambda_{L P A} \\
\text { (2) } \lambda_{E X C} \\
\text { (1) } \lambda_{L P B} \\
\text { (0) } \lambda_{G E N}\end{array}$ & $\begin{aligned}-0.1818 & \pm \mathrm{j} 298.18 \\
-0.0223 & \pm \mathrm{j} 202.70 \\
0.7875 & \pm \mathrm{j} 160.85 \\
-0.6416 & \pm \mathrm{j} 127.07 \\
-0.1097 & \pm \mathrm{j} 99.48 \\
-0.1813 & \pm \mathrm{j} 9.93\end{aligned}$ & & \\
\hline Other $\mathrm{r}$ & modes & $\begin{array}{c}-8.2345 \pm j 3072.36 \\
-8.3724 \pm j 2293.64 \\
-7.1008 \pm j 590.56 \\
-6.9133 \pm j 162.83 \\
-4.8293 \pm j 0.2969 \\
-499.97019 \\
-101.66485 \\
-32.06689 \\
-24.80366 \\
-8.11471 \\
-1.80831 \\
-3.97812 \\
-0.14183 \\
-3.27838\end{array}$ & $\begin{array}{c}-31.5121 \pm j 3036.35 \\
-926.6337 \pm j 1538.23 \\
-10.2643 \pm j 592.15 \\
-57.4064 \pm j 247.06 \\
-10.1193 \pm j 156.36 \\
-4.8552 \pm j 0.2690 \\
-499.99587 \\
-101.68570 \\
-97.73752 \\
-32.20669 \\
-24.81693 \\
-7.91593 \\
-1.75985 \\
-0.14179 \\
-3.71192 \\
-3.48712\end{array}$ & $\begin{aligned} &-31.7210 \pm j 3036.06 \\
&-926.7504 \pm j 2348.93 \\
&-10.9490 \pm j 591.49 \\
&-377.3710 \pm j 255.27 \\
&-10.4926 \pm j 159.72 \\
&-71.2491 \pm j 64.97 \\
&-6.1753 \pm j 16.71 \\
&-2.5230 \pm j 4.1863 \\
&-4.6776 \pm j 0.4270 \\
&-3.5138 \pm j 0.1098 \\
&-500.50760 \\
&-133.03238 \\
&-95.86946 \\
&-24.82959 \\
&-7.31586 \\
&-0.14179 \\
&-1.75949 \\
&-4.51796\end{aligned}$ \\
\hline
\end{tabular}

$P_{G}=0.9$ p.u.; $P F=0.9$ lagging; $X_{C A C} / X_{L A C}=50 \% ; V_{t}=1.05$ p.u.; (AC system) $a_{R}=18^{\circ}$. $y_{1}=15^{\circ}$ (ACDC system)

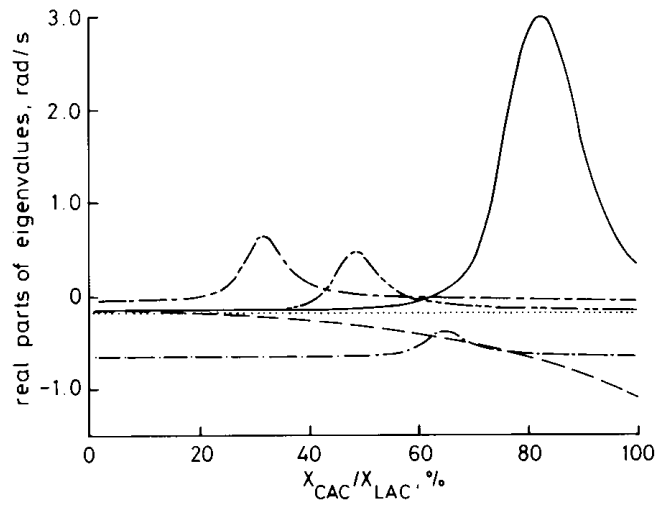

Fig. 2 Real parts of SSR mode eigenvalues as a function of $X_{C A C} / X_{L A C}(A C D C$ system without SSDC and PSS $)$

-..- mode 0

- mode 1

-. - mode 2

-.- mode 3

- - mode 4

Therefore the three SSR modes 1, 3, and 4 deserve special attention in the design of an SSDC. In addition to these SSR modes, the electromechanical mode (mode 0) should also be damped to improve its damping characteristic.

\section{Design of a supplementary subsynchronous damping controller using modal control theory}

To stabilise the three SSR modes and the electromechanical mode (mode 0 ), a compensator with two feedback signals, $\Delta \omega$ (generator speed deviation) and $\Delta I_{R}$ (rectifier current deviation), is employed to generate two supplementary damping signals, $U_{E}$ and $U_{R}$, for the exciter and the rectifier current regulator, respectively. It should be noted that the proposed compensator can be regarded as a combination of an SSDC, which generates the damping signal $U_{R}$, and a power system stabiliser (PSS), which generates the damping signal $U_{E}$. The power system stabiliser is required because the electromechanical mode (mode 0) must be controlled in addition to the three torsional modes (modes 1, 3, and 4). Fig. 3 depicts the block diagram of the proposed compensator.
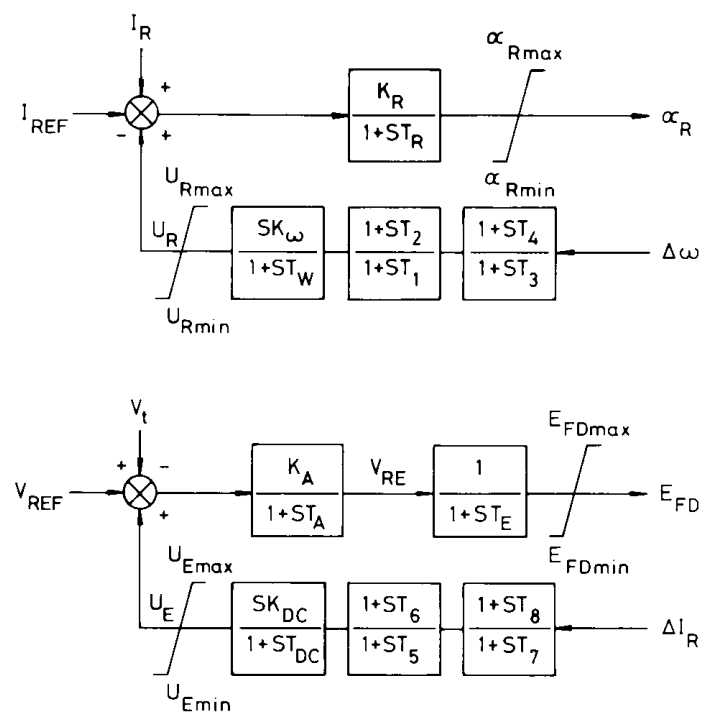

Fig. 3 Block diagram of the rectifier current regulator and excitation control system with proposed compensator (SSDC and PSS) included

In the design of the proposed compensator using modal control theory, the state equations of the system linearised at the specified operating point are first written

$$
\begin{aligned}
\dot{X}(t) & =A X(t)+B U(t) \\
Y(t) & =C(X(t)
\end{aligned}
$$

where $X(t)$ is the state vector that can be divided into two subvectors, $X_{M}$ and $X_{E}$, comprising the state variables in 
the mechanical system and electrical system, respectively.

$$
\begin{aligned}
X_{M}= & {\left[\Delta \omega_{H P}, \Delta \theta_{H P}, \Delta \omega_{I P}, \Delta \theta_{I P}, \Delta \omega_{L P A}, \Delta \theta_{I P A},\right.} \\
& \Delta \omega_{L P B}, \Delta \theta_{L P B}, \Delta \omega, \Delta \delta, \Delta \omega_{E X C}, \Delta \theta_{E X C}, \Delta T_{H P}, \\
& \left.\Delta T_{I P}, \Delta T_{L P A}, \Delta a, \Delta g\right]^{T} \\
X_{E}= & {\left[\Delta i_{d}, \Delta i_{f d}, \Delta i_{k d}, \Delta i_{q}, \Delta i_{f q}, \Delta i_{k q}, \Delta E_{F D}, \Delta V_{R E},\right.} \\
& \Delta e_{c d}, \Delta e_{c q}, \Delta i_{d A C}, \Delta i_{q A C}, \Delta V_{d A}, \Delta V_{q A}, \Delta I_{R}, \\
& \left.\Delta V_{D C}, \Delta I_{I}, \Delta \alpha_{R}, \Delta \gamma_{I}\right]^{T}
\end{aligned}
$$

The output vector can be written as

$$
Y=\left[\Delta \omega, \Delta I_{R}\right]^{T}
$$

and the input vector is

$$
U=\left[U_{E}, U_{R}\right]^{T}
$$

The open-loop transfer function in complex frequency $s$ domain from input $U$ to putput $Y$ is

$$
G(s)=C(s I-A)^{-1} B
$$

The compensator transfer functions, $H_{\omega}(s)$ and $H_{D C}(s)$, for the PSS and SSDC, respectively, are as follows [17]:

$$
\begin{gathered}
H_{\omega}(s)=\frac{s K_{\omega}}{1+s T_{\omega}}\left(\frac{1+s T_{2}}{1+s T_{1}}\right)\left(\frac{1+s T_{4}}{1+s T_{3}}\right) \\
H_{D C(s)}=\frac{s K_{D C}}{1+s T_{D C}}\left(\frac{1+s T_{6}}{1+s T_{5}}\right)\left(\frac{1+s T_{8}}{1+s T_{7}}\right)
\end{gathered}
$$

where $K_{\omega}, T_{\omega}, K_{D C}, T_{D C}$, and $T_{1}-T_{8}$ are the unknown parameters to be determined. The feedback transfer function matrix $H(s)$ is

$$
H(s)=\left[\begin{array}{cc}
H_{\omega}(s) & 0 \\
0 & H_{D C}(s)
\end{array}\right]
$$

Taking the Laplace transform of eqns. 1 and 2, we have the state equations in frequency domain

$$
\begin{aligned}
s X(s) & =A X(s)+B U(s) \\
Y(s) & =C X(s)
\end{aligned}
$$

The input signals are derived from output feedback and can be expressed as

$$
U(s)=H(s) Y(s)
$$

Substituting eqn. 11 into eqn. 9 , we have

$$
s X(s)=A X(s)+B H(s) Y(s)
$$

where $Y(s)$ can be replaced by eqn. 10 as

$$
\begin{aligned}
& s X(s)=A X(s)+B H(s) C X(s) \\
& {[s I-A-B H(s) C] X(s)=0}
\end{aligned}
$$

If $s$ is the desired eigenvalues to be located in the closedloop, $X(s)$ in eqn. 14 is not identically equal to zero. Therefore the matrix $[s I-A-B H(s) C]$ is not full rank and

$$
\operatorname{det}[s I-A-B H(s) C]=0
$$

Eqn. 15 can be factored into the product of two terms, as given by the following equation:

$$
\operatorname{det}[s I-A] \operatorname{det}\left[I-(s I-A)^{-1} B H(s) C\right]=0
$$

Consider the following matrix identity for determinants of products:

$$
\operatorname{det}[I+P Q]=\operatorname{det}[I+Q P]
$$

where $P$ and $Q$ are matrices with appropriate dimensions and $I$ is an identity matrix.
Eqn. 16 is then transformed to

$$
\operatorname{det}[s I-A] \operatorname{det}\left[I-C(s I-A)^{-1} B H(s)\right]=0
$$

Substituting eqn. 5 into eqn. 18 , the closed-loop characteristic equation is then given by

$$
\operatorname{det}[s I-A] \operatorname{det}[I-G(s) H(s)]=0
$$

where $s$ is the assigned eigenvalues placed in the closedloop. Since the desired closed-loop eigenvalues are, in general, different from the open-loop eigenvalues, which can be computed by solving the equation

$$
\operatorname{det}[s I-A]=0
$$

it follows that

$$
\operatorname{det}[I-G(s) H(s)]=0
$$

Thus, the unknown parameters of the two-input-twooutput controller can be obtained by solving a set of algebraic equations resulting from the substitution of a set of preassigned eigenvalues into eqn. 21. For the present case, four of the twelve unknown parameters must be prespecified since only eight eigenvalues associated with modes $0,1,3$, and 4 will be assigned, resulting in eight algebraic equations.

Prespecified parameters [17]

$$
T_{1}=T_{3}=T_{5}=T_{7}=0.1 \mathrm{~s}
$$

\section{Assigned eigenvalues}

$$
\begin{array}{ll}
-6.6 \pm \mathrm{j} 199.2 & \text { (mode } 4) \\
-1.1 \pm \mathrm{j} 157.4 & \text { (mode } 3) \\
-2.3 \pm \mathrm{j} 91.9 & \text { (mode } 1) \\
-3.2 \pm \mathrm{j} 10.3 & \text { (mode } 0)
\end{array}
$$

Using the above assigned eigenvalues, the following controller parameters are obtained:

$$
\begin{gathered}
K_{\omega}=-0.03, T_{\omega}=0.01 \mathrm{~s}, T_{2}=0.5 \mathrm{~s}, T_{4}=0.5 \mathrm{~s} \\
K_{D C}=-0.14, T_{D C}=0.1 \mathrm{~s}, T_{6}=0.5 \mathrm{~s}, T_{8}=0.5 \mathrm{~s}
\end{gathered}
$$

The only constraint in the selection of the assigned eigenvalues is that the resulting parameters should be reasonable (for example, the time constants should be positive).

Using the above proposed controller, the closed-loop eigenvalues under the operating conditions described above are listed in Table 2 . The real parts of the eigenvalues for modes $0-5$ as a function of $X_{C A C} / X_{L A C}$ are plotted in Fig. 4 . It can be observed that all the torsional modes will be stable for $35 \%<X_{C A C} / X_{L A C}<70 \%$.

\section{Sensitivity studies}

To demonstrate the insensitivity of the proposed SSDC, eigenvalue analyses are carried out over a wide range of operating conditions. Some results are listed in Tables 3-5. In Table $3, P_{G o}$ is fixed at 0.9 p.u., and $\alpha_{R}$ is varied from $10^{\circ}$ to $20^{\circ}$. The eigenvalues for different values of $P_{G}$ are summarised in Table 4 . Table 5 gives the results for the case with fixed $\mathrm{AC}$ line power $P_{A C}\left(P_{A C o}=\right.$ 0.4064 p.u.) but with different DC line power $P_{D C}$ (or different $\alpha_{R o}$.

It can be concluded from Tables 3-5 that, under the preassigned parameters, the proposed SSDC, although designed at a particular operating condition, can provide 
Table 3: Eigenvalues for various values of $\alpha_{R}\left(P_{G o}=0.9\right.$ p.u.)

\begin{tabular}{|c|c|c|c|c|c|c|}
\hline $\operatorname{mode}^{a_{R}}$ & $10^{\circ}$ & $12^{\circ}$ & $14^{\circ}$ & $16^{\circ}$ & $18^{\circ *}$ & $20^{\circ}$ \\
\hline $\begin{array}{l}5 \\
4 \\
3 \\
2 \\
1 \\
0\end{array}$ & $\begin{array}{l}-0.2 \pm j 298.2 \\
-9.5 \pm j 198.5 \\
-2.1 \pm j 156.5 \\
-1.0 \pm j 126.6 \\
-2.3 \pm j 92.2 \\
-1.9 \pm j 11.0\end{array}$ & $\begin{array}{l}-0.2 \pm j 298.2 \\
-8.4 \pm j 198.5 \\
-1.8 \pm j 156.5 \\
-1.0 \pm j 126.6 \\
-2.3 \pm j 92.0 \\
-2.2 \pm j 11.0\end{array}$ & $\begin{array}{l}-0.2 \pm j 298.2 \\
-7.7 \pm j 198.7 \\
-1.5 \pm j 156.7 \\
-1.0 \pm j 126.6 \\
-2.3 \pm j 91.9 \\
-2.6 \pm j 10.9\end{array}$ & $\begin{array}{l}-0.2 \pm \mathrm{j} 298.2 \\
-7.1 \pm \mathrm{j} 198.9 \\
-1.3 \pm \mathrm{j} 157.0 \\
-1.0 \pm \mathrm{j} 126.6 \\
-2.3 \pm \mathrm{j} 91.9 \\
-2.9 \pm \mathrm{j} 10.6\end{array}$ & $\begin{array}{l}-0.2 \pm j 298.2 \\
-6.6 \pm j 199.2 \\
-1.1 \pm j 157.4 \\
-1.0 \pm j 126.6 \\
-2.3 \pm j 91.9 \\
-3.2 \pm j 10.3\end{array}$ & $\begin{array}{l}-0.2 \pm j 298.2 \\
-6.1 \pm j 199.5 \\
-0.9 \pm j 157.8 \\
-1.0 \pm j 126.6 \\
-2.3 \pm j 92.0 \\
-3.3 \pm j 10.0\end{array}$ \\
\hline
\end{tabular}

- Denotes the operating point on which the SSDC design is based

Table 4: Eigenvalues for different values of $P_{G}\left(\alpha_{k o}=18^{\circ}\right)$

\begin{tabular}{|c|c|c|c|c|c|}
\hline mode $P_{G}$ & 0.7 & $0.9^{*}$ & 1.1 & 1.3 & 1.5 \\
\hline $\begin{array}{l}5 \\
4 \\
3 \\
2 \\
1 \\
0\end{array}$ & $\begin{array}{l}-0.2 \pm j 298.2 \\
-6.9 \pm j 199.8 \\
-1.1 \pm j 157.4 \\
-1.0 \pm j 126.6 \\
-2.0 \pm j 92.5 \\
-2.8 \pm j 10.5\end{array}$ & $\begin{array}{l}-0.2 \pm j 298.2 \\
-6.6 \pm j 199.2 \\
-1.1 \pm j 157.4 \\
-1.0 \pm j 126.6 \\
-2.3 \pm j 91.9 \\
-3.2 \pm j 10.3\end{array}$ & $\begin{array}{l}-0.2 \pm j 298.2 \\
-6.3 \pm j 198.8 \\
-1.0 \pm j 157.4 \\
-1.0 \pm j 126.6 \\
-2.5 \pm j 91.5 \\
-3.5 \pm j 10.1\end{array}$ & $\begin{array}{l}-0.2 \pm j 298.2 \\
-6.1 \pm j 198.6 \\
-0.8 \pm j 157.6 \\
-1.0 \pm j 126.6 \\
-2.6 \pm j 91.3 \\
-3.8 \pm j 10.0\end{array}$ & $\begin{array}{l}-0.2 \pm j 298.2 \\
-5.9 \pm j 198.8 \\
-0.6 \pm j 158.0 \\
-1.1 \pm j 126.6 \\
-2.7 \pm j 91.3 \\
-4.1 \pm j 9.8\end{array}$ \\
\hline
\end{tabular}

* Denotes the operating point on which the SSDC design is based

\begin{tabular}{|c|c|c|c|c|c|}
\hline mode $P_{D C}$ & 0.5860 & 0.5358 & $0.4846^{*}$ & 0.4323 & 0.3791 \\
\hline $\begin{array}{l}5 \\
4 \\
3 \\
2 \\
1 \\
0\end{array}$ & $\begin{array}{l}-0.2 \pm j 298.2 \\
-6.9 \pm j 198.6 \\
-1.3 \pm j 157.0 \\
-1.0 \pm j 126.6 \\
-2.4 \pm j 91.7 \\
-3.2 \pm j 10.6\end{array}$ & $\begin{array}{l}-0.2 \pm j 298.2 \\
-6.7 \pm j 198.9 \\
-1.2 \pm j 157.2 \\
-1.0 \pm j 126.6 \\
-2.4 \pm j 91.8 \\
-3.2 \pm j 10.5\end{array}$ & $\begin{array}{l}-0.2 \pm j 298.2 \\
-6.6 \pm j 199.2 \\
-1.1 \pm j 157.4 \\
-1.0 \pm j 126.6 \\
-2.3 \pm j 91.9 \\
-3.2 \pm j 10.3\end{array}$ & $\begin{array}{l}-0.2 \pm \mathrm{j} 298.2 \\
-6.4 \pm \mathrm{j} 199.4 \\
-1.0 \pm \mathrm{j} 157.6 \\
-1.0 \pm \mathrm{j} 126.6 \\
-2.2 \pm \mathrm{j} 92.1 \\
-3.2 \pm \mathrm{j} 10.2\end{array}$ & $\begin{array}{l}-0.2 \pm j 298.2 \\
-6.3 \pm j 199.7 \\
-0.9 \pm j 157.8 \\
-1.0 \pm j 126.6 \\
-2.1 \pm j 92.2 \\
-3.2 \pm j 10.1\end{array}$ \\
\hline
\end{tabular}

* Denotes the operating point on which the SSDC design is based

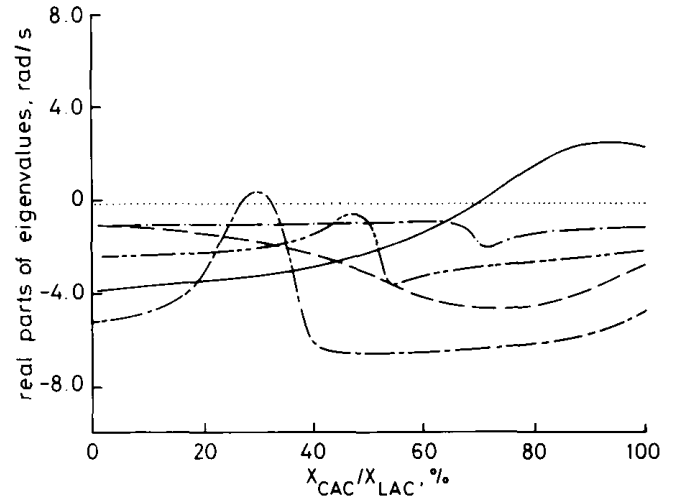

Fig. 4 Real parts of SSR mode eigenvalues as a function of $X_{C A C} / X_{L A C}(A C D C$ system with SSDC and PSS)

-... mode 0

- mode

-. - mode 2

-.... mode

- - mode 4

satisfactory damping characteristic over a wide range of operating conditions.

\section{Nonlinear simulations}

To illustrate the effectiveness of the proposed SSDC which is designed based on a linearised system, under disturbance conditions, time domain simulations are performed using a nonlinear system model including all system nonlinearities such as exciter ceilings, control signal limiters etc.

The dynamic responses to a 0.2 p.u. step disturbance in generator torque $\left(\Delta T_{G E N}\right)$, which lasts for four cycles $(0.067 \mathrm{~s})$, are shown in Figs. 5 and 6 for the system without and with an SSDC, respectively. The response curves in Fig. 5 indicate the expected unstable nature of the open-loop system and those in Fig. 6 clearly demonstrate the effectiveness of the SSDC in providing positive dampings for the SSR modes under disturbance conditions. The required control signals for the PSS and SSDC, $U_{E}$ and $U_{R}$, are plotted in Fig. 7. It is observed that only moderate signals are needed for damping purposes.

\section{Conclusions}

A systematic approach based on modal control theory is presented for the design of a supplementary subsynchronous damping controller (SSDC) equipped at the rectifier end of an HV DC link connected in parallel with an AC line. The subsynchronous oscillations on the series-compensated AC line, as well as the torsional interaction between the HV DC converter and turbine generator, can be effectively damped by the proposed SSDC. Sensitivity studies indicate that the proposed controller is insensitive in that it can provide good damping effect over a wide range of operating conditions. Digital simulations based on a nonlinear model are also performed to demonstrate the effectiveness of the proposed controller under major disturbance conditions.

IEE PROCEEDINGS, Vol. 136, Pt. C, No. 2, MARCH 1989 

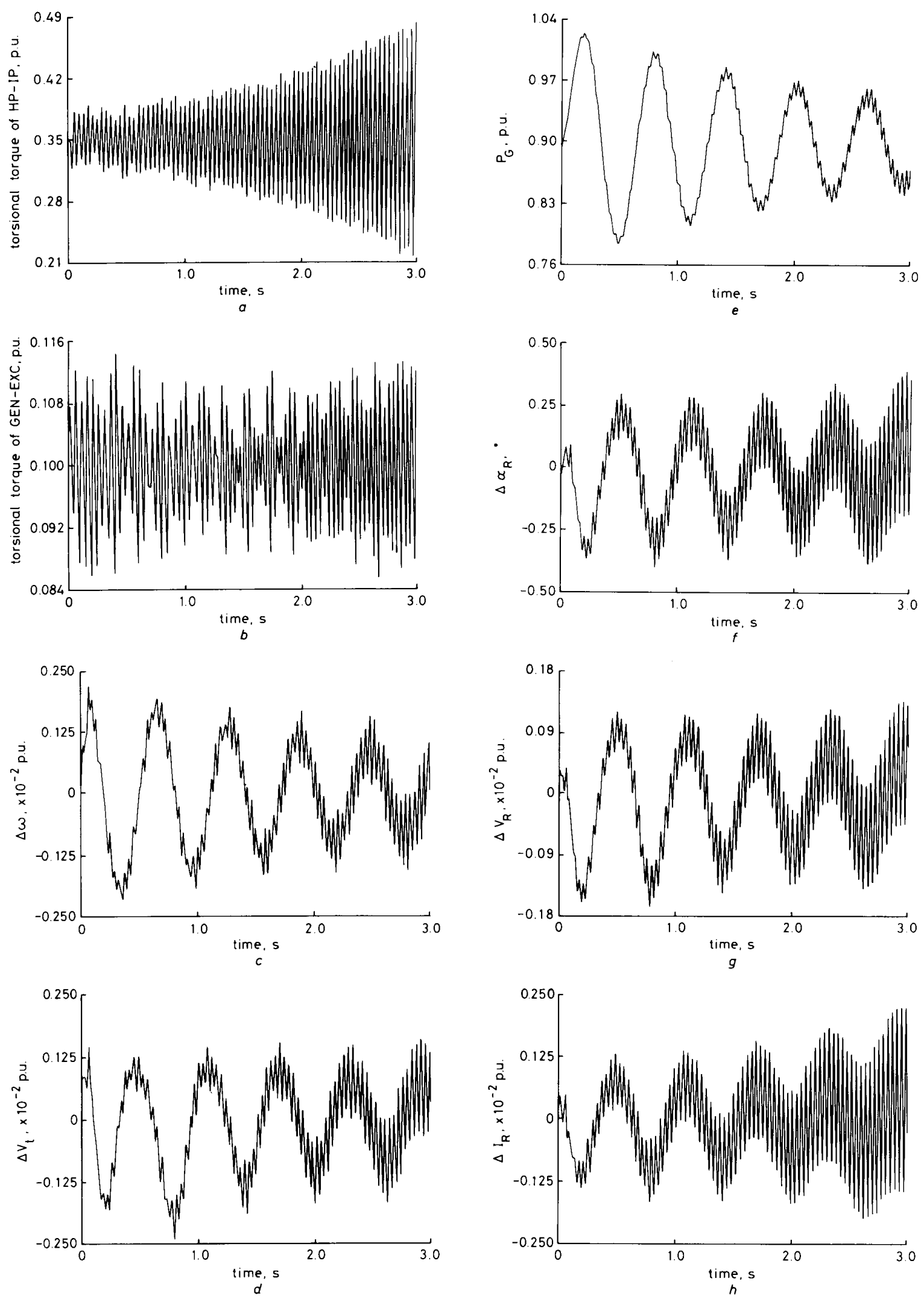

Fig. 5 Dynamic responses for the system without SSDC and PSS

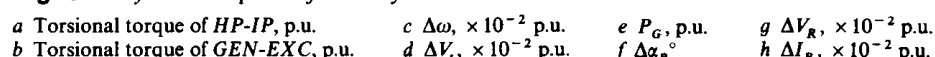

IEE PROCEEDINGS, Vol. 136, Pt.C, No. 2, MARCH 1989 

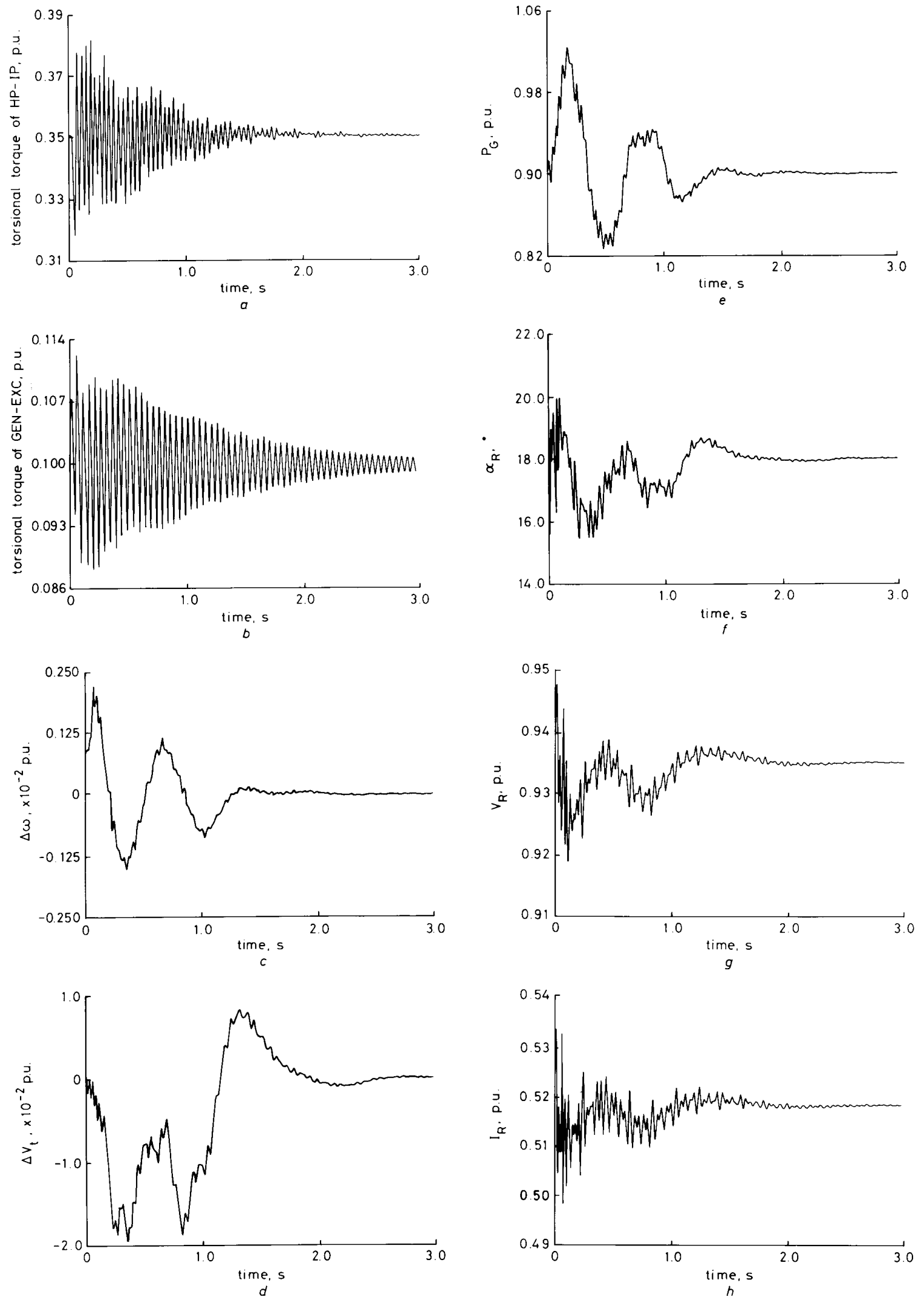

Fig. 6 Dynamic responses for the system with SSDC and PSS a Torsional torque of $H P-I P$, p.u. $\quad c \Delta \omega, \times 10^{-2}$ p.u. $\quad$ e $P_{G}$, p.u. $\quad g V_{R}$, p.u 84 

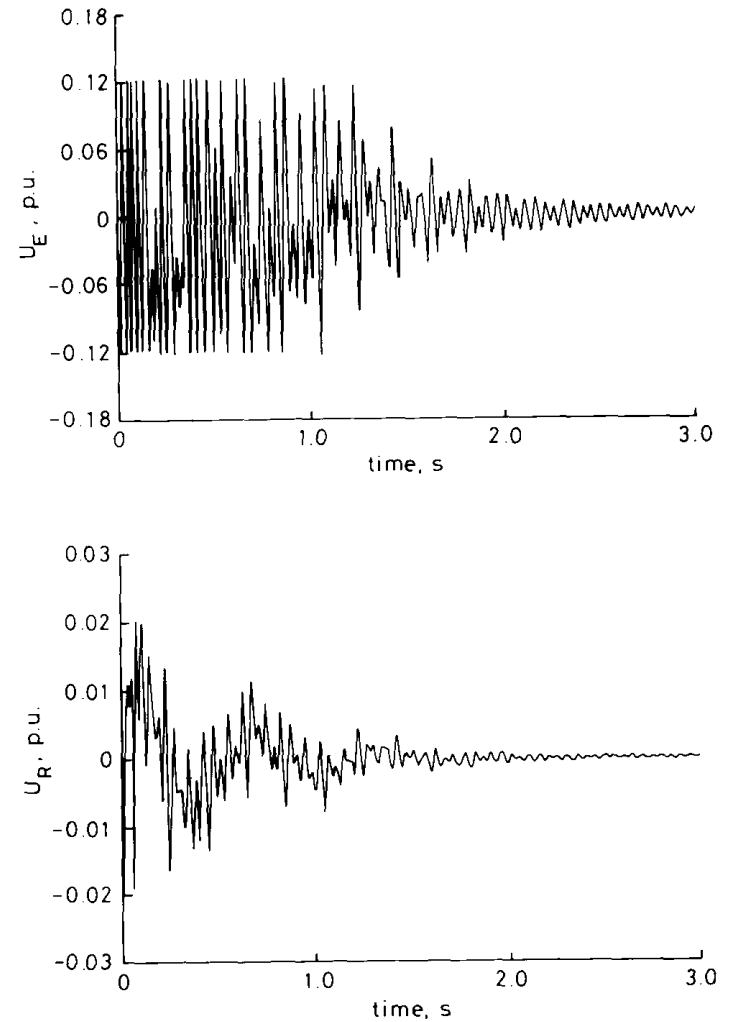

Fig. 7 Control signals for the PSS and SSDC

$a$ PSS, $U_{E}$
$b$ SSDC, $U_{*}$

\section{References}

1 BAHRMAN, M. LARSEN, E.V PIWKO, R.J., and PATEL, H.S. 'Experience with HV DC-turbine-generator torsional interaction at Square Butte', IEEE Trans., 1980, PAS-99, pp. 966-975

2 BJORKLUND, H., JOHANSSON, K.E., and LISS, G.: 'Damping of subsynchronous oscillations in systems containing turbine generators and HV DC links'. CIGRE Paper 14-01, 1980

3 BAHRMAN, M.P., LARSEN, E.V., PIWKO, R.J., PATEL, H.S., HAUTH, R.L., and BREUER, G.D.: 'HV DC-turbine-generator torsional interactions: a new design consideration'. CIGRE Paper $14-04,1980$

4 MORTENSEN, K., LARSEN, E.V., and PIWKO, R.J.: 'Field tests and analysis of torsional interaction between the Coal Creek turbine-generators and the CU HV DC system', IEEE Trans., 1981, PAS-100, pp. 336-344

5 SVENSSON, S., and MORTENSEN, K.: 'Damping of subsynchronous oscillations by an HV DC link: An HV DC simulator study', IEEE Trans., 1981, PAS-100, pp. 1431-1439

6 PIWKO, R.J., and LARSEN, E.V.: 'HV DC system control for damping of subsynchronous oscillations'. EPRI Report EL-2708, 1982

7 HARLEY, R.G., and BALDA, J.C.: 'Subsynchronous resonance damping by specially controlling a parallel HV DC link', IEE Proc C, Gen., Trans. \& Distrib., 1985, 132, pp. 154-160

8 PORTER, B., and CROSSLEY, R.: 'Modal control theory and applications' (Taylor \& Francis, 1972)

9 CHEN, C.L., and HSU, Y.Y.: 'Coordinated synthesis of multimachine power system stabilizer using an efficient decentralized modal control (DMC) algorithm'. Paper 86 SM 321-4, presented at the IEEE/PES 1986 Summer Meeting, Mexico City

10 IEEE Committee Report: 'First benchmark model for computer simulation of subsynchronous resonance'. IEEE Trans., 1977, PAS-96, pp. 1565-1572

11 PETERSON, H.A., and KRAUSE, P.C.: 'A direct- and quadratureaxis representation of a parallel $\mathrm{AC}$ and $\mathrm{DC}$ power system', IEEE Trans., 1966, PAS-86, pp. 210-224

12 YU, Y.N.: 'Electric power system dynamics' (Academic Press, New York, 1983)

IEE PROCEEDINGS, Vol. 136, Pt. C, No. 2, MARCH 1989
13 KIMBARK, E.W.: 'Direct current transmission' (Wiley, New York, 1971)

14 RAHIM, A.H.M.A., and EL-AMIN, I.M.: 'Stabilization of a high voltage AC/DC power system I. Evaluation of control strategies', IEEE Trans., 1985, PAS-104, pp. 3084-3090

15 CHOUDHRY, M.A., EMARAH, A.S., ELLITHY, K.A., and GALANOS, G.D.: 'Stability analysis of a modulated AC/DC system using the eigenvalue sensitivity approach', IEEE Trans., 1986, PWRS-1, pp. 128-136

16 ANDERSON, P.M., and FOUAD, A.A.: 'Power system control and stability' (Iowa State University Press, Ames, lowa, 1977)

17 LARSEN, E.V., and SWANN, D.A.: 'Applying power system stabilizers', IEEE Trans., 1981, PAS-100, pp. 3017-3046

18 IEEE Committee: 'Dynamic models for steam and hydro turbines in power system studies', IEEE Trans., 1973, PAS-92, pp. 1904-1915

\section{Appendixes}

\subsection{DC transmission line equations}

All the equations given in the Appendix are in per unit. The base values for the $\mathrm{AC}$ and DC quantities should be chosen such that the per unit values of any DC quantities will remain unchanged when converted to the reference frame of AC systems [13]. Thus, the DC quantities of rectifier output voltages and currents can be converted with reference to the common frame of the $d q$-axis of AC systems, according to the phasor diagram in Fig. 8 [11].

$$
\begin{aligned}
& V_{q R}=V_{q} \cos \delta_{R}+V_{d} \sin \delta_{R} \\
& V_{d R}=-V_{q} \sin \delta_{R}+V_{d} \cos \delta_{R} \\
& I_{q R}=I_{q} \cos \delta_{R}+I_{d} \sin \delta_{R} \\
& I_{d R}=-I_{q} \sin \delta_{R}+I_{d} \cos \delta_{R}
\end{aligned}
$$

where $\delta_{R}$ is the angle by which $q_{R}$-axis lags the $q$-axis.

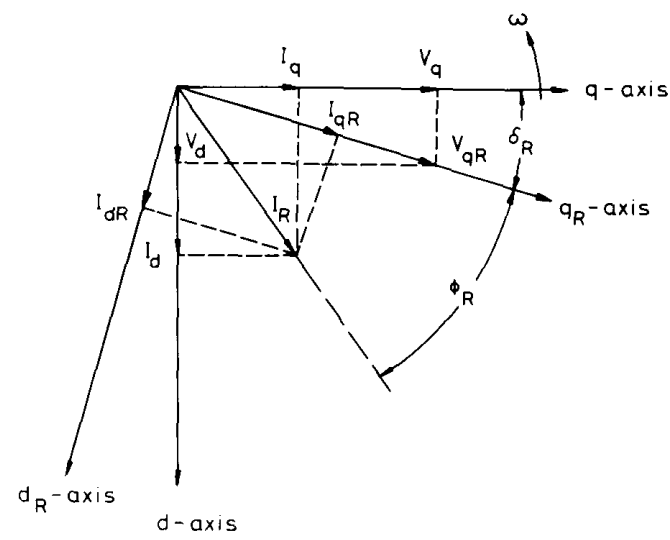

Fig. 8 Phasor diagram for the dq quantities

Since the reference direction for the $q_{R}$-axis with respect to $q$-axis is arbitrary, it is convenient to select a direction which makes $V_{d R}$ vanish. Although transformation selection is unnecessary to restrict $V_{d R}$ to zero, this particular selection allows the operation of the converter to be easily expressed. Under this reference frame, we have the following relationships:

$$
\begin{aligned}
\cos \delta_{R} & =V_{q} / V_{q R} \\
\sin \delta_{R} & =V_{d} / V_{q R} \\
I_{d} & =I_{d R} \cos \delta_{R}+I_{q R} \sin \delta_{R}
\end{aligned}
$$

and

$$
I_{q}=-I_{d R} \sin \delta_{R}+I_{q R} \cos \delta_{R}
$$


The rectifier voltage, $V_{R}$, and inverter voltage, $V_{I}$, can be written as [14]

$$
\begin{gathered}
V_{R}=V_{q R} \cos \alpha_{R}-\frac{\pi}{6} X_{C R} I_{R} \\
V_{I}=V_{q I} \cos \gamma_{I}-\frac{\pi}{6} X_{C I} I_{I}
\end{gathered}
$$

where $V_{q I}=V_{\infty}$, as shown in Fig. 1. Thus, the $d R$-axis and $q R$-axis components of $I_{R}$ are given by

$$
\begin{aligned}
& I_{q R}=I_{R} \sin \phi_{R} \\
& I_{d R}=I_{R} \cos \phi_{R}
\end{aligned}
$$

respectively, where the power factor is defined by

$$
\cos \phi_{R}=V_{R} / V_{q R}
$$

The equations for the rectifier and inverter regulators are [14]

$$
\begin{aligned}
& \dot{\alpha}_{R}=\left(\frac{K_{R}}{T_{R}}\right)\left(-I_{R E F}+I_{R}+U_{R}\right)-\left(\frac{1}{T_{R}}\right) \alpha_{R} \\
& \dot{\gamma}_{I}=\left(\frac{K_{I}}{T_{I}}\right)\left(K_{m} I_{R E F}-I_{I}\right)-\left(\frac{1}{T_{I}}\right) \gamma_{I}
\end{aligned}
$$

It is noted that the rectifier and inverter are both under constant current control. The steady-state operating point is found by first choosing a pair of values for $\alpha_{R}$ and $\gamma_{I}$ and then solving the network equations to find the rectifier current, $I_{R}$, and inverter current, $I_{I}$.
8.2 Turbine torques and speed governor system

A two-time-constant governor is used in this paper, and two differential equations for the governor are [12]

$$
\begin{aligned}
& \dot{a}=\left(\frac{K_{G}}{T_{S R}}\right)\left(\omega_{R E F}-\omega\right)-\left(\frac{1}{T_{S R}}\right) a \\
& \dot{g}=\frac{1}{T_{S M}}(a-g)
\end{aligned}
$$

where $T_{S R}$ and $T_{S M}$ are, respectively, the time constants of the speed relay and the servomotor. The units for $\omega, a$ and $g$ in eqns. 37 and 38 are all in per unit values.

All turbine torques are proportional to contribute the net mechanical torque for the mass-spring system. It is assumed that the exciter electrical torque output $T_{E X C}$ is zero. The equations for system turbine torques are as follows $[12,18]$ :

$$
\begin{aligned}
\dot{T}_{H P} & =\frac{1}{T_{C H}}\left(F_{H P} g-T_{H P}\right) \\
\dot{T}_{I P} & =\frac{1}{T_{R H}}\left(\frac{F_{I P}}{F_{H P}} T_{H P}-T_{I P}\right) \\
\dot{T}_{L P A} & =\frac{1}{T_{C O}}\left(\frac{F_{L P A}}{F_{I P}} T_{I P}-T_{L P A}\right) \\
T_{L P B} & =\frac{F_{L P B}}{F_{L P A}} T_{L P A}
\end{aligned}
$$

where $T_{C H}, T_{R H}$ and $T_{C O}$ are the time constants of the steam chest in front of the high pressure turbine, the reheater between high and medium-pressure turbines, and the crossover connection between the medium and low pressure turbines, respectively. 